\title{
Disturbance Attenuation of Uncertain Nonholonomic Systems in Chained Forms
}

\author{
Yi Guo \\ Department of Electrical and Computer Engineering \\ Stevens Institute of Technology, Hoboken, NJ 07030, USA \\ yguo1@stevens.edu
}

\begin{abstract}
Nonlinear $H_{\infty}$ control is considered for uncertain nonholonomic systems with external disturbances in their chained form kinematic models. State feedback controllers are explicitly constructed to guarantee an $\mathcal{L}_{2}$ gain performance from the disturbance to the system output. Without the presence of the disturbance, the system states are regulated to the origin. Recent results in robust and adaptive control of uncertain nonholonomic systems are extended to include external disturbance inputs. An simulation example shows the effectiveness of the proposed control schemes.
\end{abstract}

Index Terms-Nonlinear control, nonholonomic systems, backstepping, disturbance attenuation, $\mathcal{L}_{2}$ gain.

\section{INTRODUCTION}

There has been increasing interest in studying uncertain robotic systems with nonholonomic constrains for the last decade due to its inherent nonlinearity and challenges in constructing analytic control solutions, see [13] for a good review on the control problems, and [19] for a review on both holonomic and nonholonomic constrained systems. Stabilization problem was recently solved for uncertain kinematic model in its chained form in [11]. The adaptive version of a similar configuration was presented in [7]. Near optimal tracking control was designed in [18] to take into account explicitly the control effort required to solve a given problem. Combining the kinematic model with the dynamic model in a cascaded form, stabilization and tracking controls for uncertain nonholonomic systems were designed in [6], [5], [4], [16]. It seems that disturbance attenuation problem has not been covered much in existing literatures. Exceptions include [3] (and some references therein), where the authors consider model reference control for perturbed nonholonomic systems with external disturbance, and an $H_{\infty}$ performance was derived at the last stage of the design.

In this paper, we consider disturbance attenuation for uncertain nonholonomic kinematic systems in their chained form models. Nonlinear $H_{\infty}$ control problem is solved for such systems. Since the initial results proposed in [17], nonlinear $H_{\infty}$ control was discussed intensively for structured systems in [15], [9], [10], and constructive techniques using backstepping were exploited. Later on, decentralized nonlinear $H_{\infty}$ control was discussed in [8], [12]. Since disturbance is a commonly existing component in nonholonomic mechanic system dynamics, this paper presents new results in designing $H_{\infty}$ controller for uncertain nonholonoimc systems with external disturbance inputs. Explicit state feedback controllers are constructed so that the effect of the disturbance on the system output is attenuated to any given level in the sense of an $\mathcal{L}_{2}$ gain measurement. The states of the closed-loop system are regulated to the origin without the presence of disturbances. Simulation results on an example system will be given to demonstrate the responses of our controlled system.

\section{Class of Systems And Control Problem}

As stated in the Introduction, many nonlinear mechanical systems with nonholonomic constraints can be transformed to a canonical chained form representation. We consider a class of the uncertain nonholonomic systems in their perturbed chained form:

$$
\begin{aligned}
\dot{x}_{0}= & u_{0}+\gamma_{0}\left(t, x_{0}\right)+p_{0}^{T}(t) \omega \\
\dot{x}_{i}= & u_{0} x_{i+1}+\gamma_{i}\left(t, x_{0}, x_{1}, \ldots, x_{i}, u_{0}\right) \\
& +p_{i}^{T}\left(t, x_{0}, x_{1}, \ldots, x_{i}, u_{0}\right) \omega \quad 1 \leq i<n \\
\dot{x}_{n}= & u_{1}+\gamma_{n}\left(t, x_{0}, x_{1}, \ldots, x_{n}, u_{0}\right) \\
& +p_{n}^{T}\left(t, x_{0}, x_{1}, \ldots, x_{n}, u_{0}\right) \omega \\
y= & x_{1}
\end{aligned}
$$

where $x=\left(x_{0}, x_{1}, \ldots, x_{n}\right) \in \Re^{n+1}$ are the system states, $u_{0}, u_{1} \in \Re$ are the system input, $\omega \in \Re^{m}$ is the disturbance input, $y \in \Re$ is the output, $\gamma_{i}, p_{i}, i=0,1, \ldots, n$ are unknown functions/vectors and are locally Lipschitz in states and piecewise continuous in $t$, and $\gamma_{i}(t, 0, \ldots, 0)=0$.

Assumption 1: There exist positive constants $k_{1}, k_{2}$ such that

$$
\begin{aligned}
\left|\gamma_{0}\left(t, x_{0}\right)\right| & \leq k_{1}\left|x_{0}\right| \\
\left|p_{0}(t)\right| & \leq k_{2} .
\end{aligned}
$$

Assumption 2: There exist known smooth nonnegative functions $\phi_{i}, \psi_{i}(1 \leq i \leq n)$, and a positive constant $\psi_{i 0}$ such that

$$
\begin{aligned}
& \left|\gamma_{i}\left(t, x_{0}, x_{1}, \ldots, x_{i}, u_{0}\right)\right| \leq\left|\left(x_{1}, \ldots, x_{i}\right)\right| \\
& \quad \cdot \phi_{i}\left(x_{0}, x_{1}, \ldots, x_{i}, u_{0}\right) \\
& \left|p_{i}\left(t, x_{0}, x_{1}, \ldots, x_{i}, u_{0}\right)\right| \leq\left|\left(x_{1}, \ldots, x_{i}\right)\right| \\
& \cdot \psi_{i}\left(x_{0}, x_{1}, \ldots, x_{i}, u_{0}\right)+\psi_{i 0} .
\end{aligned}
$$

The objective of our design is to find state feedback controllers to make the closed-loop system uniformly asymptotically stable while arbitrarily attenuating the effect of the disturbance in the sense of an $\mathcal{L}_{2}$ gain. A precise statement of this control problem is given below: 
Problem of $H_{\infty}$ Almost Disturbance Decoupling: Find state feedback controllers $u_{0}\left(x_{0}\right), u_{1}(x)$ such that, for any given positive constant $\mu$, the closed-loop interconnected system satisfies the following dissipation inequality

$$
\begin{aligned}
& \int_{0}^{\infty}|y(t)|^{2} d t \leq \mu \int_{0}^{\infty}|\omega(t)|^{2} d t+\nu(x(0)), \\
& \forall \omega(t) \in \mathcal{L}_{2}
\end{aligned}
$$

where $\nu$ is a positive semi-definite function and $x(0)$ is the initial condition. Furthermore, uncertain system (2) is regulated to the origin if $\omega=0$.

\section{State Feedback Controller Design}

\section{A. Design $u_{0}$ for the $x_{0}$-Subsystem}

We first design $u_{0}$ for the $x_{0}$-subsystem. The following lemmas will be used in our controller design.

Lemma 1 ([14]): Given a differentiable function $f(t)$ : $\Re^{+} \rightarrow \Re$, if $f \in \mathcal{L}_{2}$ and $\dot{f} \in \mathcal{L}_{2}$, then $f \rightarrow 0$ and $f \in \mathcal{L}_{\infty}$.

Lemma 2 ([14]): Given a differentiable function $f(t)$ : $\Re^{+} \rightarrow \Re$, if $f_{1} \in \mathcal{L}_{2}$ and $f_{2} \in \mathcal{L}_{2}$, then $f_{1}+f_{2} \in \mathcal{L}_{2}$.

Consider the following positive definite function

$$
V_{0}=x_{0}^{2}+\int_{t}^{\infty} \omega^{T} \omega d t, \quad \forall T \geq t
$$

Note that $V_{0}$ is not a Lyapunov function since the system may not have an equilibrium point.

Since $\omega \in \mathcal{L}_{2}$, there exists a constant $C$ such that

$$
\int_{0}^{t} \omega^{T} \omega d t+\int_{t}^{\infty} \omega^{T} \omega d t=C<\infty
$$

Taking the derivative with respect to time, we obtain

$$
\omega^{T} \omega+\frac{d}{d t}\left[\int_{t}^{\infty} \omega^{T} \omega d t\right]=0
$$

From equations (7), (9), (1), and (4) in Assumption 1, we have

$$
\begin{aligned}
\dot{V}_{0} & =2 x_{0}\left[u_{0}+\gamma_{0}\left(t, x_{0}\right)+p_{0}^{T}(t) \omega\right]-\omega^{T} \omega \\
& \leq 2 x_{0} u_{0}+2 k_{1}\left|x_{0}\right|^{2}+2 k_{2}\left|x_{0}\right||\omega|-\omega^{T} \omega
\end{aligned}
$$

Using the inequality

$$
2 a b \leq a^{2}+b^{2},(a, b \in \Re)
$$

to the third term, we get

$$
\begin{aligned}
\dot{V}_{0} \leq & 2 x_{0} u_{0}+2 k_{1}\left|x_{0}\right|^{2}+k_{2}^{2}\left|x_{0}\right|^{2} \\
& +|\omega|^{2}-\omega^{T} \omega
\end{aligned}
$$

Choose

$$
u_{0}=-\left(c_{0}+k_{1}+\frac{1}{2} k_{2}^{2}\right) x_{0} \triangleq \lambda_{0} x_{0}
$$

where $c_{0}$ is a positive constant. Then (12) turns to:

$$
\dot{V}_{0} \leq-2 c_{0} x_{0}^{2}
$$

Therefore $V_{0}$ is a non-increasing function and thus $V_{0} \in \mathcal{L}_{\infty}$ which implies that $x_{0} \in \mathcal{L}_{\infty}$. Integrating (14), we get $x_{0} \in$ $\mathcal{L}_{2}$. From (4) in Assumption 1 and Lemma 2, we conclude that $\dot{x} \in \mathcal{L}_{2}$. Finally from Lemma 1 , since $x, \dot{x} \in \mathcal{L}_{2}$, we get $x \rightarrow 0$.

\section{B. State Scaling}

From the above analysis, we see that by choosing $u_{0}$ as in (13), the $x_{0}$ state in (1) can be regulated to zero as $t \rightarrow \infty$. Since the system (2) is un-controllable in the limit $u_{0}=0$, discontinuous coordinate transformation is needed to avoid the un-controllable situation, as used in [2], [11], [7]. The following discontinuous coordinate transformation, an application of $\sigma$ process ([1]), is defined as the following:

$$
z_{i}=\frac{x_{i}}{x_{0}^{n-i}}, \quad 1 \leq i \leq n
$$

In the new $z$-coordinates, the system (2) is transformed into

$$
\begin{aligned}
\dot{z}_{i}= & \lambda_{0} z_{i+1}+f_{i}\left(t, x_{0}, z_{1}, \ldots, z_{i}\right) \\
& +g_{i}\left(t, x_{0}, z_{1}, \ldots, z_{i}\right) \omega, \quad 1 \leq i<n \\
\dot{z}_{n}= & u_{1}+f_{n}\left(t, x_{0}, z_{1}, \ldots, z_{n}\right) \\
& +g_{n}\left(t, x_{0}, z_{1}, \ldots, z_{n}\right) \omega
\end{aligned}
$$

where for $1 \leq i \leq n$,

$$
\begin{aligned}
f_{i}\left(x_{0}, z_{1}, \ldots, z_{i}\right)= & -(n-i) \lambda_{0} z_{i}-\frac{(n-i) z_{i}}{x_{0}} \gamma_{0} \\
& +\frac{1}{x_{0}^{n-i}} \gamma_{i} \\
g_{i}\left(x_{0}, z_{1}, \ldots, z_{i}\right)= & -\frac{(n-i) z_{i}}{x_{0}} p_{0}^{T}+\frac{1}{x_{0}^{n-i}} p_{i}^{T}
\end{aligned}
$$

It is not difficult to obtain the following conditions on $f_{i}, g_{i}, \quad 1 \leq i \leq n$, which are analogous with those in Assumption 2:

$$
\begin{aligned}
\left|f_{i}\left(t, x_{0}, z_{1}, \ldots, z_{i}\right)\right| \leq & \left|\left(z_{1}, \ldots, z_{i}\right)\right| \\
& \bar{\phi}_{i}\left(x_{0}, z_{1}, \ldots, z_{i}\right) \\
\left|g_{i}\left(t, x_{0}, z_{1}, \ldots, z_{i}\right)\right| \leq & \left|\left(z_{1}, \ldots, z_{i}\right)\right| \\
& \bar{\psi}_{i}\left(x_{0}, z_{1}, \ldots, z_{i}\right)+\bar{\psi}_{i 0}\left(x_{0}\right)
\end{aligned}
$$

where $\bar{\phi}_{i}, \bar{\psi}_{i}$ are smooth nonnegative functions.

\section{Backstepping Design for $u_{1}$}

In this subsection, we apply recursive backstepping design procedure to the dynamics (16) in the $z$-coordinates.

Step 1: From (16), the $z_{1}$-subsystem dynamics is:

$$
\dot{z}_{1}=\lambda_{0} z_{2}+f_{1}\left(x_{0}, z_{1}\right)+g_{1}\left(x_{0}, z_{1}\right) \omega .
$$

To design a virtual control $z_{2}=z_{2}^{*}\left(x_{0}, z_{1}\right)$ for (19), we define a storage function $V_{1}$ as

$$
V_{1}\left(z_{1}\right)=z_{1}^{2} .
$$

Taking time derivative of $V_{1}$, we get:

$$
\begin{aligned}
\dot{V}_{1} \leq & 2 z_{1} \lambda_{0} z_{2}+2\left|z_{1}\right|^{2} \bar{\phi}_{1}\left(x_{0}, z_{1}\right) \\
& +2\left|z_{1}\right|^{2} \bar{\psi}_{1}\left(x_{0}, z_{1}\right)|\omega|+2\left|z_{1}\right| \bar{\psi}_{10}\left(x_{0}\right)|\omega|
\end{aligned}
$$


Using (11) to the last term in the above equation, we get

$$
\begin{aligned}
\dot{V}_{1} \leq & \frac{1}{d_{11}}\left|z_{1}\right|^{4} \bar{\psi}_{1}^{2}\left(x_{0}, z_{1}\right)+\frac{1}{d_{12}}\left|z_{1}\right|^{2} \bar{\psi}_{10}^{2}\left(x_{0}\right) \\
& +\left(d_{11}+d_{12}\right)|\omega|^{2}
\end{aligned}
$$

where $d_{11}, d_{12}$ are positive design constants.

Choosing the virtual control $z_{2}^{*}$ as

$$
\begin{gathered}
z_{2}^{*}\left(x_{0}, z_{1}\right)=\frac{1}{\lambda_{0}}\left[-\frac{c_{11}}{2} z_{1}-z_{1} \bar{\phi}_{1}\left(x_{0}, z_{1}\right)\right. \\
\left.-\frac{1}{2 d_{11}} z_{1}^{3} \bar{\psi}_{1}^{2}\left(x_{0}, z_{1}\right)-\frac{1}{2 d_{12}} z_{1} \bar{\psi}_{10}^{2}\left(x_{0}\right)\right]
\end{gathered}
$$

where $c_{11}$ is positive constant to be chosen later. Then we have

$$
\dot{V}_{1} \leq-c_{11} z_{1}^{2}+d_{1}|\omega|^{2}+2 z_{1} \lambda_{0}\left(z_{2}-z_{2}^{*}\right)
$$

where $d_{1}=d_{11}+d_{12}$.

It is easy to check that $z_{2}^{*}\left(x_{0}, z_{1}\right)$ is a smooth function, and $z_{2}^{*}\left(x_{0}, 0\right)=0, \frac{\partial z_{2}^{*}}{\partial x_{0}}\left(x_{0}, 0\right)=0$.

Step 2: Augment the $z_{2}$ subsystem to the $z_{1}$-subsystem, and choose a storage function

$$
V_{2}\left(z_{1}, z_{2}\right)=V_{1}\left(z_{1}\right)+\left(z_{2}-z_{2}^{*}\right)^{2}
$$

Differentiating $V_{2}$ along the $z_{1}, z_{2}$ dynamics, we get

$$
\dot{V}_{2}=\dot{V}_{1}+2\left(z_{2}-z_{2}^{*}\right)\left(\lambda_{0} z_{3}+f_{2}+g_{2} \omega-\dot{z}_{2}^{*}\right)
$$

Note that

$$
\begin{aligned}
\dot{z}_{2}^{*}= & \frac{\partial z_{2}^{*}}{\partial x_{0}} \dot{x}_{0}+\frac{\partial z_{2}^{*}}{\partial z_{1}} \dot{z}_{1} \\
= & \frac{\partial z_{2}^{*}}{\partial z_{1}} \cdot \lambda_{0} z_{2}+\left[\frac{\partial z_{2}^{*}}{\partial x_{0}} \cdot\left(\lambda_{0} x_{0}+\gamma_{0}\right)+\frac{\partial z_{2}^{*}}{\partial z_{1}} \cdot f_{1}\right] \\
& +\left(\frac{\partial z_{2}^{*}}{\partial x_{0}} \cdot p_{0}^{T}+\frac{\partial z_{2}^{*}}{\partial z_{1}} \cdot g_{1}\right) \omega
\end{aligned}
$$

Substitute (27) into (26), we get

$$
\begin{aligned}
\dot{V}_{2} \leq & -c_{11} z_{1}^{2}+d_{1}|\omega|^{2}+2\left(z_{2}-z_{2}^{*}\right)\left(\lambda_{0} z_{3}\right. \\
& \left.+\lambda_{0} z_{1}-\frac{\partial z_{2}^{*}}{\partial z_{1}} \cdot \lambda_{0} z_{2}-\frac{\partial z_{2}^{*}}{\partial x_{0}} \lambda_{0} x_{0}\right) \\
& +\Delta_{2}\left(x_{0}, z_{1}, z_{2}\right)+\eta_{2}\left(x_{0}, z_{1}, z_{2}\right) \omega
\end{aligned}
$$

where

$$
\begin{aligned}
& \Delta_{2}=2\left(z_{2}-z_{2}^{*}\right)\left(-\frac{\partial z_{2}^{*}}{\partial x_{0}} \gamma_{0}-\frac{\partial z_{2}^{*}}{\partial z_{1}} f_{1}+f_{2}\right) \\
& \eta_{2}=2\left(z_{2}-z_{2}^{*}\right)\left(-\frac{\partial z_{2}^{*}}{\partial x_{0}} p_{0}^{T}-\frac{\partial z_{2}^{*}}{\partial z_{1}} g_{1}+g_{2}\right)
\end{aligned}
$$

Applying (4), (18), (27) and (11) to the uncertain term $\Delta_{2}$, and after lengthy but simple calculations, there exist smooth nonnegative function $\vartheta_{21}, \vartheta_{22}$ such that:

$$
\begin{aligned}
\left|\Delta_{2}\right| & \leq \widetilde{z}_{2}^{2} \vartheta_{21}\left(x_{0}, z_{1}, z_{2}\right)+l_{21} z_{1}^{2} \\
\left|\eta_{2} \omega\right| & \leq \widetilde{z}_{2}^{2} \vartheta_{22}\left(x_{0}, z_{1}, z_{2}\right)+d_{21}|\omega|^{2}
\end{aligned}
$$

where $l_{21}, l_{22}, d_{21}$ are positive constants, $\widetilde{z}_{2}=z_{2}-z_{2}^{*}$, and

$$
\begin{array}{ll}
\vartheta_{21}\left(x_{0}, 0,0\right)=0, & \frac{\partial \vartheta_{21}}{\partial x_{0}}\left(x_{0}, 0,0\right)=0 . \\
\vartheta_{22}\left(x_{0}, 0,0\right)=0, & \frac{\partial \vartheta_{22}}{\partial x_{0}}\left(x_{0}, 0,0\right)=0 .
\end{array}
$$

Choose the virtual control $z_{3}^{*}\left(x_{0}, z_{1}, z_{2}\right)$ as the following:

$$
\begin{aligned}
z_{3}^{*}= & -\frac{c_{22}}{2} \widetilde{z}_{2}-z_{1}+\frac{\partial z_{2}^{*}}{\partial z_{1}} z_{2}+\frac{\partial z_{2}^{*}}{\partial x_{0}} x_{0} \\
& -\frac{1}{2 \lambda_{0}} \widetilde{z}_{2}\left[\vartheta_{21}\left(x_{0}, z_{1}, z_{2}\right)+\vartheta_{22}\left(x_{0}, z_{1}, z_{2}\right)\right]
\end{aligned}
$$

where $c_{22}$ is a positive design constant. It can be checked that

$$
z_{3}^{*}\left(x_{0}, 0,0\right)=0, \quad \frac{\partial z_{3}^{*}}{\partial x_{0}}\left(x_{0}, 0,0\right)=0 .
$$

Then we have

$$
\begin{aligned}
\dot{V}_{2} \leq & -c_{21} z_{1}^{2}-c_{22} \widetilde{z}_{2}^{2}+d_{2}|\omega|^{2} \\
& +2 \lambda_{0} \widetilde{z}_{2}\left(z_{3}-z_{3}^{*}\right)
\end{aligned}
$$

where

$$
c_{21}=c_{11}-l_{21}, \quad d_{2}=d_{1}+d_{21} .
$$

Step $i(3 \leq i \leq n-1)$ : Assume that from Step $i-1$, we have designed a virtual control $z_{i}^{*}\left(x_{0}, z_{1}, \ldots, z_{i-1}\right)$, so that for the chosen storage function $V_{i-1}$, which has the property

$$
z_{i}^{*}\left(x_{0}, 0, \ldots, 0\right)=0 \quad \frac{\partial z_{i}^{*}}{\partial x_{0}}\left(x_{0}, 0, \ldots, 0\right)=0 .
$$

For the chosen storage function $V_{i-1}$, its time derivative is

$$
\dot{V}_{i-1} \leq \sum_{j=1}^{i-1}-c_{i-1, j} \widetilde{z}_{j}^{2}+d_{i-1}|\omega|^{2}+2 \lambda_{0} \widetilde{z}_{i-1} \widetilde{z}_{i}(37)
$$

where $\widetilde{z}_{1}=z_{1}, \widetilde{z}_{j}=z_{j}-z_{j}^{*}, 1<j \leq n-1$. Choose

$$
V_{i}=V_{i-1}+\left(z_{i}-z_{i}^{*}\right)^{2}
$$

Its time derivative is

$$
\begin{aligned}
\dot{V}_{i} \leq & \sum_{j=1}^{i-1}-c_{i-1, j} \widetilde{z}_{j}^{2}+d_{i-1}|\omega|^{2}+2 \widetilde{z}_{i}\left\{\lambda_{0} \widetilde{z}_{i-1}\right. \\
& \left.+\lambda_{0} z_{i+1}-\frac{\partial z_{i}^{*}}{\partial x_{0}} \lambda_{0} x_{0}-\sum_{j=1}^{i-1} \frac{\partial z_{i}^{*}}{\partial z_{j}} \lambda_{0} z_{j+1}\right\} \\
& +\Delta_{i}+\eta_{i} \omega
\end{aligned}
$$

where

$$
\begin{aligned}
\Delta_{i} & =2 \widetilde{z}_{i}\left(-\frac{\partial z_{i}^{*}}{\partial x_{0}} \gamma_{0}-\sum_{j=1}^{i-1} \frac{\partial z_{i}^{*}}{\partial z_{j}} f_{j}+f_{i}\right) \\
\eta_{i} & =2 \widetilde{z}_{i}\left(-\frac{\partial z_{i}^{*}}{\partial x_{0}} p_{0}^{T}-\sum_{j=1}^{i-1} \frac{\partial z_{i}^{*}}{\partial z_{j}} g_{j}+g_{i}\right)
\end{aligned}
$$


Applying bounds to the two uncertain terms in the above equation, and after some calculations, we get:

$$
\begin{aligned}
\left|\Delta_{i}\right| & \leq \sum_{j=1}^{i-1} l_{i 1} \widetilde{z}_{j}^{2}+\widetilde{z}_{i}^{2} \vartheta_{i 1}\left(x_{0}, z_{1}, \ldots, z_{i}\right) \\
\left|\eta_{i} \omega\right| & \leq \widetilde{z}_{i}^{2} \vartheta_{i 2}\left(x_{0}, z_{1}, \ldots, z_{i}\right)+d_{i 1}|\omega|^{2}
\end{aligned}
$$

where $l_{i 1}, l_{i 2}, d_{i 1}$ are positive constants, $\vartheta_{i 1}, \vartheta_{i 2}$ are smooth nonnegative functions and have the same property as in (31).

Choose virtual control as

$$
\begin{aligned}
z_{i+1}^{*}= & -\frac{c_{i i}}{2} \widetilde{z}_{i}-z_{i-1}+\frac{\partial z_{i}^{*}}{\partial x_{0}} x_{0}+\sum_{j=1}^{i-1} \frac{\partial z_{i}^{*}}{\partial z_{j}} z_{j+1} \\
& -\frac{1}{2 \lambda_{0}} \widetilde{z}_{i}\left[\vartheta_{i 1}+\vartheta_{i 2}\right]
\end{aligned}
$$

Substitute (41) and (42) into (39), we obtain:

$$
\dot{V}_{i} \leq \sum_{j=1}^{i}-c_{i j} \widetilde{z}_{j}^{2}+d_{i}|\omega|^{2}+2 \lambda_{0} \widetilde{z}_{i} \widetilde{z}_{i+1}
$$

where

$$
c_{i j}=c_{i-1, j}-l_{i 1}, j<i \quad d_{i}=d_{i-1}+d_{i 1}
$$

Step $n$ : In this step, we design the true control $u_{1}$ for the whole system. Choose the storage function

$$
V_{n}=V_{n-1}+\widetilde{z}_{n}^{2}
$$

where $\widetilde{z}_{n}=z_{n}-z_{n}^{*}$.

Its time derivative along the system dynamics (16) is

$$
\begin{aligned}
\dot{V}_{n} \leq & \sum_{j=1}^{n-1}-c_{n-1, j}{\widetilde{z_{j}}}^{2}+d_{n-1}|\omega|^{2}+2 \widetilde{z}_{n}\left\{\lambda_{0} \widetilde{z}_{n-1}\right. \\
& \left.+u_{1}-\frac{\partial z_{n}^{*}}{\partial x_{0}} \lambda_{0} x_{0}-\sum_{j=1}^{n-1} \frac{\partial z_{n}^{*}}{\partial z_{j}} \lambda_{0} z_{j+1}\right\} \\
& +\Delta_{n}+\eta_{n} \omega
\end{aligned}
$$

where

$$
\begin{aligned}
& \Delta_{n}=2 \widetilde{z}_{n}\left(-\frac{\partial z_{n}^{*}}{\partial x_{0}} \gamma_{0}-\sum_{j=1}^{n-1} \frac{\partial z_{n}^{*}}{\partial z_{j}} f_{j}+f_{n}\right) \\
& \eta_{n}=2 \widetilde{z}_{n}\left(-\frac{\partial z_{n}^{*}}{\partial x_{0}} p_{0}^{T}-\sum_{j=1}^{n-1} \frac{\partial z_{n}^{*}}{\partial z_{j}} g_{j}+g_{n}\right)
\end{aligned}
$$

As in the previous steps, we can find smooth nonnegative functions $\vartheta_{n 1}, \vartheta_{n 2}$ such that:

$$
\begin{aligned}
& \left|\Delta_{n}\right| \leq \sum_{j=1}^{n-1} l_{n 1} \widetilde{z}_{j}^{2}+\widetilde{z}_{n}^{2} \vartheta_{n 1}\left(x_{0}, z_{1}, \ldots, z_{n}\right) \\
& \left|\eta_{n} \omega\right| \leq \widetilde{z}_{n}^{2} \vartheta_{n 2}\left(x_{0}, z_{1}, \ldots, z_{n}\right)+d_{n 1}|\omega|^{2}
\end{aligned}
$$

where $l_{n 1}, l_{n 2}, d_{n 1}$ are positive constants.
Our true control $u_{1}$ is designed to be:

$$
\begin{aligned}
u_{1}= & -\frac{c_{n n}}{2} \widetilde{z}_{n}-\lambda_{0} z_{n-1}+\lambda_{0} \frac{\partial z_{n}^{*}}{\partial x_{0}} x_{0} \\
& +\lambda_{0} \sum_{j=1}^{n-1} \frac{\partial z_{n}^{*}}{\partial z_{j}} z_{j+1}-\frac{1}{2} \widetilde{z}_{n}\left[\vartheta_{n 1}+\vartheta_{n 2}\right]
\end{aligned}
$$

Substitute (47) and (48) into (45), we obtain:

$$
\dot{V}_{n} \leq \sum_{j=1}^{n}-c_{n j}{\widetilde{z_{j}}}^{2}+d_{n}|\omega|^{2}
$$

where

$$
c_{n j}=c_{n-1, j}-l_{n 1}, j<n d_{n}=d_{n-1}+d_{n 1}
$$

\section{Switching Strategy}

In the case of $x_{0}=0$, we need to design a switching controller to avoid singularity of the state scaling (15). It has been pointed out in [11], [7] that for uncertain terms that do not satisfy the Lipschitz condition, the states may blow up within a finite time. Following similar ideas, we choose a different $u_{0}$ when $x_{0}=0$ :

$$
u_{0}=\lambda_{0} x_{0}+\bar{C}
$$

where $\bar{C}$ is any positive constant. Applying it to the time derivative of $V_{0}$ in (7), we obtain

$$
\dot{V}_{0} \leq-2 c_{0} x_{0}^{2}+\bar{C} x_{0},
$$

from which we can conclude the boundedness of $x_{0}$.

For the stability of states $x_{1}, \ldots, x_{n}$, replace $u_{0}$ by (50) (instead of (13)) in the system dynamics (2). And then applying the same backstepping design procedure to the new $z$-coordinate dynamics as described above, we will get an essentially same inequality as (49), which implies that states $x_{1}, \ldots, x_{n}$ do not blow up.

\section{E. Main Theorem}

We are now ready to present our main result:

Theorem 1: For the system (1) (3) under Assumptions 1 and 2, the control laws (13), (48) and the switching strategy presented above solve the Problem of $H_{\infty}$ Almost Disturbance Decoupling.

Proof: We know that the state $x_{0}$ is regulated to 0 as $t \rightarrow \infty$ from Section III-A. In the $z$-coordinates, from the last step in the recursive backstepping design, we obtained (49). If we choose design parameters $l_{i 1}, l_{i 2}, c_{i j}, 1 \leq i, j \leq n, j \leq$ $i$ such that $c_{n j}>0$, when $\omega=0$, we have a positive definite and radially unbounded Lyapunov function (44), and its time derivative is negative definite. Therefore we can conclude that $\widetilde{z}_{i}, 1 \leq i \leq n$ are uniformly asymptotically stable (UAS). Since $\widetilde{z}_{i}=z_{i}-z_{i}^{*}$, and $z_{i}^{*}\left(x_{0}, 0, \ldots, 0\right)=0$, we get that $z_{i}$ is UAS, which implies UAS of states $x$ in the original coordinates.

When $\omega \neq 0$, taking the integral of (49) along time $t$, we can obtain

$$
\int_{0}^{\infty}|y(t)|^{2} d t \leq \mu \int_{0}^{\infty}|\omega(t)|^{2} d t+\nu(\widetilde{z}(0))
$$



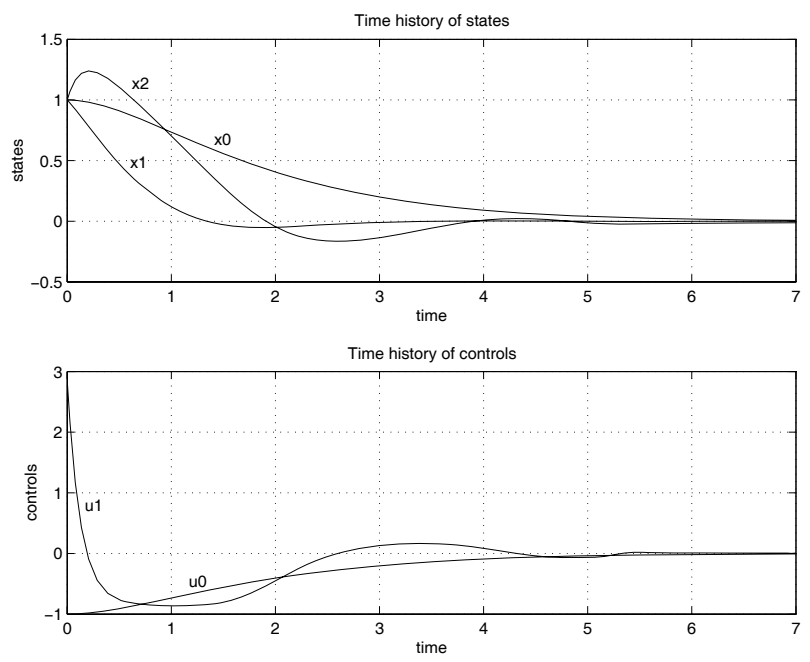

Fig. 1. Closed-loop responses and control input history.

where

$$
\mu=d_{n} / c_{n 1} \nu(\widetilde{z}(0))=\sum_{i=1}^{n}\left(\widetilde{z}_{i}(0)\right)^{2} .
$$

This complete the proof of Theorem 1.

\section{A Simulation Example}

We consider the following example system which belongs to the class of systems in this paper's interest:

$$
\begin{aligned}
& \dot{x}_{0}=u_{0}+0.1 \omega \\
& \dot{x}_{1}=x_{2} u_{0}+d_{1}(t) x_{1}^{2}+d_{2}(t) \omega \\
& \dot{x}_{2}=u_{1}+d_{3}(t) x_{1} \omega
\end{aligned}
$$

where

$$
\begin{gathered}
d_{1}(t)=0.1 \sin (t), d_{2}(t)=0.1 \cos (0.5 t), \\
d_{3}(t)=0.2, \omega=e^{-t} .
\end{gathered}
$$

We choose $u_{0}=-x_{0}$ for the $x_{0}$-subsystem, and apply the state scaling

$$
z_{1}=\frac{x_{1}}{x_{0}}, z_{2}=x_{2} \text {. }
$$

Following the design procedure showed in Steps 1 and 2 of Section III-C, the responses of the closed-loop system and the time history of control inputs are shown in Figure 1. It can be seen that our control scheme achieves satisfactory performances.

\section{CONCLUSIONS}

In this paper, we consider nonlinear $H_{\infty}$ control for a class of uncertain nonholonomic systems in their chained forms. Recent results presented in [11], [7] are extended to the class of uncertain chained form systems with external disturbances. Constructive controllers are designed using backstepping, and the so-called $H_{\infty}$ almost disturbance decoupling problem is solved. The states of the closedloop system are regulated to the origin without the presence of disturbances. With the presence of the disturbance, the effect of the disturbance on the system output is attenuated to any given level in the sense of an $\mathcal{L}_{2}$ gain measurement. An simulation example shows the effectiveness of our control schemes.

\section{REFERENCES}

[1] V. I. Arnold. Geometrical methods in the theory of ordinary differential equations. Springer, Berlin, 1987.

[2] A. Astolfi. Discontinuous control of nonholonomic systems. Systems and Control Letters, 27:37-45, 1996.

[3] B. S. Chen, T. S.Lee, and W. S. Chang. A robust $H_{\infty}$ model reference tracking design for non-holonomic mechanical control systems. Int. J. Control, 63(2):283-306, 1996.

[4] W. Dong. On trajectory and force tracking control of constrained mobile manipulators with parameter uncertainty. Automatica, 38:14751484, 2002.

[5] W. Dong and W. L. Xu. Adaptive tracking control of uncertain nonholonomic dynamic systems. IEEE Trans. Automat. Contr., 46(3):450-454, 2001.

[6] W. Dong, Y. Xu, and W. Huo. On stabilization of uncertain dynamic nonholonomic systems. Int. J. Control, 73(4):349-359, 2000.

[7] S. S. Ge, Z. Wang, and T. H. Lee. Adaptive stabilization of uncertain nonholonomic systems by state and output feedback. Automatica, 39:1451-1460, 2003.

[8] Y. Guo, Z. P. Jiang, and D. J. Hill. Decentralized robust disturbance attenuation for a class of large-scale nonlinear systems. Systems and Control Letters, 37:71-85, 1999.

[9] A. Isidori. A note on almost disturbance decoupling for nonlinear minimum phase systems. Systems and Control Letters, 27:191-194, 1996.

[10] D. C. Jiang and Z. P. Jiang. $H_{\infty}$ almost disturbance decoupling with stability for uncertain nonlinear systems. In Proc. 4th European Control Conf., Brussels, Belgium, 1997.

[11] Z. P. Jiang. Robust exponential regulation of nonholonomic systems with uncertainties. Automatica, 36:189-209, 2000.

[12] Z. P. Jiang, D. W. Repperger, and D. J. Hill. Decentralized nonlinear output-feedback stablization with disturbance attenuation. IEEE Trans. Automat. Contr., 46(10):1623-1629, 2001.

[13] I. Kolmanovsk and N. H. McClamroch. Developments in nonholonomic control sytems. IEEE Control Systems Magzine, 15(6):20-36, 1995

[14] R. Lozano, B. Brogliato, O. Egeland, and B. Maschke. Dissipative Systems Analysis and Control: Theory and Applications. Communications and Control Engineering. Springer, London, 2000.

[15] R. Marino, W. Respondek, A. J. van der Schaft, and P. Tomei. Nonlinear $H_{\infty}$ almost disturbance decoupling. Systems and Control Letters, 23:159-168, 1994.

[16] M. Oya, C. Su, and R. Katoh. Robust adaptive motion/force tracking control of uncertain nonholonomic mechanical systems. IEEE Trans. Robotics and Automation, 19(1):175-181, 2003.

[17] A. J. van der Schaft. $L_{2}$-gain analysis of nonlinear systems and nonlinear state feedback $H_{\infty}$ control. IEEE Trans. Automat. Contr., 37:770-784, 1992.

[18] J. Wang and Z. Qu. A near optimal tracking control of dynamic nonholonomic systems. In IEEE International Conference on Robotics, Intelligent Systems and Signal Processing, Hunan, China, May 2003.

[19] X. Yun and N. Sarkar. Unified formulation of robotic systems with holonomic and nonholonomic constraints. IEEE Trans. Robotics and Automation, 14(4):640-650, 1998. 